\title{
Refinements of some bounds related to the constant $e$
}

\author{
Cristinel Mortici
}




\title{
REFINEMENTS OF SOME BOUNDS RELATED TO THE CONSTANT $e$
}

\author{
CRISTINEL MORTICI
}

Received 16 September, 2010

\begin{abstract}
The aim of this paper is to refine some inequalities related to the constant $e$. Finally, a strengthened Carleman's inequality is given.
\end{abstract}

2000 Mathematics Subject Classification: 33B15; 26D15

Keywords: completely monotonicity, inequalities, sharp constants, rate of convergence

\section{INTRODUCTION}

There exists an extensive literature on inequalities involving the constant $e$ and the exponential function in general. Recently, some authors have obtained interesting inequalities of that type and applied them to refine the Hardy and Carleman inequality [1]. It states that for every $a_{n} \geq 0$ with $0<\sum_{n=1}^{\infty} a_{n}<\infty$,

$$
\sum_{n=1}^{\infty}\left(a_{1} a_{2} \cdots a_{n}\right)^{1 / n}<e \sum_{n=1}^{\infty} a_{n} .
$$

One of the first contributions in this sense was achieved by Kloosterman [2, Chapter 3.8.26], who proved the following inequality

$$
e-\left(1+\frac{1}{n}\right)^{n}<\frac{e}{2 n}, \quad n=1,2,3, \ldots
$$

In the same direction, Yang [7] and Yang and Debnath [8] proved that for every $x \geq 1$,

$$
e\left(1-\frac{1}{2 x+1}\right)<\left(1+\frac{1}{x}\right)^{x}<e\left(1-\frac{1}{2 x+2}\right)
$$

respectively

$$
e\left(1-\frac{1}{2 x+\frac{5}{3}}\right)<\left(1+\frac{1}{x}\right)^{x}<e\left(1-\frac{1}{2 x+2}\right)
$$


Furthermore Zitian and Yibing [9] improved (1.2)-(1.3), showing that

$$
e\left(1-\frac{7}{14 x+12}\right)<\left(1+\frac{1}{x}\right)^{x}<e\left(1-\frac{6}{12 x+11}\right) \text {. }
$$

As an application of the inequalities (1.1)-(1.4), they obtained certain strengthened Carleman - Hardy's inequalities, due to the fact that

$$
\sum_{n=1}^{\infty}\left(a_{1} a_{2} \cdots a_{n}\right)^{1 / n}<\sum_{n=1}^{\infty}\left(1+\frac{1}{n}\right)^{n} a_{n}
$$

See [1, Chapter 9.12]. More precisely, Yang [7] applied (1.2)-(1.3) to (1.5) to establish the following improvement of Carleman's inequality

$$
\sum_{n=1}^{\infty}\left(a_{1} a_{2} \cdots a_{n}\right)^{1 / n}<e \sum_{n=1}^{\infty}\left(1-\frac{1}{2 n+2}\right) a_{n} .
$$

We show in this paper that the right-hand side of (1.4) is sharp in a sense that we explain later. The upper bound of (1.4) defines the positive function

$$
f(x)=1+\ln \left(1-\frac{6}{12 x+11}\right)-x \ln \left(1+\frac{1}{x}\right), \quad x \in(0, \infty)
$$

and in the first part of this paper we prove that $f$ is completely monotonic.

As numerical computations show, for large values of $x$, the quantity $\left(1+\frac{1}{x}\right)^{x}$ becomes closer to the right-hand side of (1.4). Motivated by this fact, we prove that the best approximation of the form

$$
\left(1+\frac{1}{x}\right)^{x} \approx e\left(1-\frac{1}{a x+b}\right), \text { as } x \rightarrow \infty
$$

is obtained indeed for $a=2$ and $b=\frac{11}{6}$. In order to strengthen our assertions, we prove that for every $x \geq 1$,

$$
e\left(1-\frac{6}{12 x+11-\frac{5}{12 x}}\right)<\left(1+\frac{1}{x}\right)^{x}<e\left(1-\frac{6}{12 x+11-\frac{5}{12 x}+\frac{17}{90 x^{2}}}\right) .
$$

\section{A COMPLETELY MONOTONICITY RESULT}

The theory of completely monotonic functions has remarkable applications in pure mathematics, and in other branches of science, such as probability, numerical and asymptotic analysis, applied statistics, or statistical physics. A collection of the most important properties of completely monotonic functions can be found in the monograph of Widder [6].

We recall that a function $z:(0, \infty) \rightarrow \mathbb{R}$ is said to be completely monotonic, if $z$ has derivatives of all orders and satisfies $(-1)^{n} z^{(n)}(x) \geq 0$ for every $n=0,1,2, \ldots$ and $x \in(0, \infty)$. 
The famous Bernstein's theorem, e.g. [6, p. 161], states that $z$ is completely monotonic if and only if

$$
z(x)=\int_{0}^{\infty} e^{-t x} d \mu(t),
$$

where $\mu$ is a nonnegative measure on $[0, \infty)$ such that the integral converges for all $x>0$. Completely monotonic functions $z$ are of great importance because they provide sharp bounds for $z$ and their derivatives.

We first prove the following

Lemma 1. Let $a_{n}=12^{n}-12 n\left(7^{n-1}-1\right)$. Then $a_{n}>0$, for every $n \geq 4$.

Proof. By the binomial formula, we have

$$
\begin{aligned}
& 12^{n}=(7+5)^{n}>7^{n}+\left(\begin{array}{c}
n \\
1
\end{array}\right) 5 \cdot 7^{n-1}+\left(\begin{array}{c}
n \\
2
\end{array}\right) 5^{2} \cdot 7^{n-2} \\
= & \left(\frac{25}{14} n^{2}+\frac{45}{14} n+7\right) 7^{n-1}>12 n \cdot 7^{n-1}>12 n\left(7^{n-1}-1\right),
\end{aligned}
$$

where the penultimate inequality follows from letting $n=s+4$, with $s \geq 0$, and

$$
\left.\left(\left(\frac{25}{14} n^{2}+\frac{45}{14} n+7\right)-12 n\right)\right|_{n=s+4}=\frac{25}{14} s^{2}+\frac{11}{2} s+\frac{3}{7}>0 .
$$

Now we are in the position to state the main result of this section. We also use the following integral representations, for every $x \in(0, \infty)$,

$$
\frac{1}{x}=\int_{0}^{\infty} e^{-x t} d t, \frac{1}{x^{2}}=\int_{0}^{\infty} t e^{-x t} d t
$$

Theorem 1. The function $f:(0, \infty) \rightarrow \mathbb{R}$ given by

$$
f(x)=1+\ln \left(1-\frac{6}{12 x+11}\right)-x \ln \left(1+\frac{1}{x}\right), \quad x \in(0, \infty)
$$

is completely monotonic.

Proof. We have

$$
f^{\prime \prime}(x)=\frac{1}{x}-\frac{1}{x+1}-\frac{1}{(x+1)^{2}}-\frac{1}{\left(x+\frac{5}{12}\right)^{2}}+\frac{1}{\left(x+\frac{11}{12}\right)^{2}}
$$

and using (2.1), we obtain

$$
\begin{aligned}
f^{\prime \prime}(x)= & \int_{0}^{\infty} e^{-x t} d t-\int_{0}^{\infty} e^{-(x+1) t} d t-\int_{0}^{\infty} t e^{-(x+1) t} d t \\
& -\int_{0}^{\infty} t e^{-\left(x+\frac{5}{12}\right) t} d t+\int_{0}^{\infty} t e^{-\left(x+\frac{11}{12}\right) t} d t,
\end{aligned}
$$


or

$$
f^{\prime \prime}(x)=\int_{0}^{\infty} \varphi(t) e^{-(x+1) t} d t,
$$

where $\varphi(t)=e^{t}-1-t-t e^{\frac{7}{12} t}+t e^{\frac{1}{12} t}$. As

$$
\varphi(12 t)=\sum_{n=4}^{\infty} \frac{a_{n}}{n !} t^{n}>0,
$$

it follows from (2.2) that $f^{\prime \prime}$ is completely monotonic. As a consequence, for every $n=2,3, \ldots$ and $x \in(0, \infty)$,

$$
(-1)^{n} f^{(n)}(x) \geq 0 .
$$

Further, $f^{\prime}$ is strictly increasing, since $f^{\prime \prime}>0$ and $f^{\prime}(\infty)=0$, so $f^{\prime}<0$. Now $f$ is strictly decreasing, with $f(\infty)=0$, so $f>0$. Finally, (2.3) also holds for $n=0,1$, thus $f$ is completely monotonic.

\section{A CLASS OF APPROXIMATIONS}

Motivated by the lower and upper approximations (1.4), we wonder whether there are real parameters $a, b$ which provide the best approximation of the form

$$
\left(1+\frac{1}{x}\right)^{x} \approx e\left(1-\frac{1}{a x+b}\right), \text { as } x \rightarrow \infty .
$$

One way to estimate the accuracy of such an approximation is to introduce the relative error sequence $w_{n}=w_{n}(a, b)$ defined by the relations

$$
\left(1+\frac{1}{n}\right)^{n}=e\left(1-\frac{1}{a n+b}\right) \exp w_{n}, \quad n \geq 1
$$

and to consider an approximation of the form (3.1) to be better, when the sequence $\left(w_{n}\right)_{n \geq 1}$ converges faster to zero. Further, a powerful tool for estimating the rate of convergence is the following

Lemma 2. If $\left(\omega_{n}\right)_{n \geq 1}$ is convergent to zero and there exists the limit

$$
\lim _{n \rightarrow \infty} n^{k}\left(\omega_{n}-\omega_{n+1}\right)=l \in \mathbb{R},
$$

with $k>1$, then there exists the limit:

$$
\lim _{n \rightarrow \infty} n^{k-1} \omega_{n}=\frac{l}{k-1}
$$

We see from Lemma 2 that the rate of convergence of the sequence $\left(\omega_{n}\right)_{n \geq 1}$ increases together with the value $k$ satisfying (3.3). This lemma was used by Mortici [5]-[4] for constructing asymptotic expansions, or to accelerate the convergence of certain sequences. For proof and some details, see, e.g., [5], or [3]. 
By (3.2), and by using a computer software, such as Maple, we get

$$
w_{n}-w_{n+1}=\left(\frac{1}{a}-\frac{1}{2}\right) \frac{1}{n^{2}}+\left(\frac{7}{6}-\frac{1}{a}+\frac{1-2 b}{a^{2}}\right) \frac{1}{n^{3}}+O\left(\frac{1}{n^{4}}\right) .
$$

Now we can see that the fastest sequence $w_{n}-w_{n+1}$, and consequently, by Lemma 2 , the fastest sequence $w_{n}$ are obtained when the first two coefficients of the power series (3.4) vanish, namely for $a=2, b=\frac{11}{6}$. In this case of the approximation is

$$
\left(1+\frac{1}{x}\right)^{x} \approx e\left(1-\frac{1}{2 x+\frac{11}{6}}\right)
$$

the relative error sequence $w_{n}\left(2, \frac{11}{6}\right)$ converges to zero as $n^{-3}$, since

$$
\lim _{n \rightarrow \infty} n^{3} w_{n}\left(2, \frac{11}{6}\right)=-\frac{5}{288}
$$

Further we have the idea to refine the class of approximations (3.5) to

$$
\left(1+\frac{1}{x}\right)^{x} \approx e\left(1-\frac{1}{2 x+\frac{11}{6}+\frac{c}{x}}\right),
$$

where $c$ is real parameter. In order to find the best value of $c$, we consider the corresponding relative error sequence $z_{n}=z_{n}(c)$ by the relations

$$
\left(1+\frac{1}{n}\right)^{n}=e\left(1-\frac{1}{2 n+\frac{11}{6}+\frac{c}{n}}\right) \exp z_{n}, \quad n \geq 1 .
$$

Making appeal again to the Maple software, we get

$$
z_{n}-z_{n+1}=\left(-\frac{3}{4} c-\frac{5}{96}\right) \frac{1}{n^{4}}+O\left(\frac{1}{n^{5}}\right) .
$$

Using again Lemma 2, the best value of $c$ is obtained when the coefficient of $n^{-4}$ vanishes, that is $c=-\frac{5}{72}$. In consequence, the best approximation of the form (3.6) is

$$
\left(1+\frac{1}{x}\right)^{x} \approx e\left(1-\frac{1}{2 x+\frac{11}{6}-\frac{5}{72 x}}\right),
$$

whose relative error sequence $z_{n}\left(-\frac{5}{72}\right)$ converges to zero as $n^{-4}$, since

$$
\lim _{n \rightarrow \infty} n^{4} z_{n}\left(-\frac{5}{72}\right)=\frac{17}{2160} \text {. }
$$

Now we can see that the sequence $z_{n}\left(-\frac{5}{72}\right)$ has a better rate of convergence than $w_{n}\left(2, \frac{11}{6}\right)$ has, and this is another evidence of the fact that the approximation (3.7) is more accurate than (3.5), for large values of $x$. 


\section{SOME SHARP BOUNDS}

Our new approach presented in the previous section can be used to establish increasingly accurate approximations. Moreover, these approximations can be used to deduce some sharp bounds, as it is done in the following theorem. Remark that the expression in the right-hand side of (4.1) was obtained using the method presented above.

Theorem 2. For every real number $x \geq 1$, we have

$$
e\left(1-\frac{6}{12 x+11-\frac{5}{12 x}}\right)<\left(1+\frac{1}{x}\right)^{x}<e\left(1-\frac{6}{12 x+11-\frac{5}{12 x}+\frac{17}{90 x^{2}}}\right) \text {. }
$$

Proof. We have to prove that $g>0$ and $h<0$, where

$$
g(x)=x \ln \left(1+\frac{1}{x}\right)-\ln \left(1-\frac{6}{12 x+11-\frac{5}{12 x}}\right)-1
$$

and

$$
h(x)=x \ln \left(1+\frac{1}{x}\right)-\ln \left(1-\frac{6}{12 x+11-\frac{5}{12 x}+\frac{17}{90 x^{2}}}\right)-1 .
$$

We have $g>0$ and $h<0$ on $[1, \infty)$, since

$$
\begin{gathered}
g^{\prime \prime}(x)=\frac{P(x)}{x(x+1)^{2}\left(132 x+144 x^{2}-5\right)^{2}\left(60 x+144 x^{2}-5\right)^{2}} \\
=-\frac{h^{\prime \prime}(x)}{x(x+1)^{2}\left(900 x^{2}-75 x+2160 x^{3}+34\right)^{2}\left(1980 x^{2}-75 x+2160 x^{3}+34\right)^{2}},
\end{gathered}
$$

where

$$
\begin{gathered}
P(x)=2210400 x^{2}-297600 x+58821120 x^{3}+123690240 x^{4} \\
+67682304 x^{5}-625 \\
Q(x)=282884760 x^{2}-96687840 x+6264927720 x^{3}+30001770225 x^{4} \\
+284790556800 x^{5}+2911276663200 x^{6}+5519218176000 x^{7} \\
+2875292640000 x^{8}+1336336 .
\end{gathered}
$$

(the polynomial $P(x+1)$ has all coefficients positive). Now $g$ is strictly convex, $h$ is strictly concave, with $g(\infty)=h(\infty)=0$, so $g>0$ and $h<0$.

We further refine Yang's result (1.6) using (1.5) and Theorem 2. 
Theorem 3. For every $a_{n} \geq 0, n=1,2,3, \ldots$, with $0<\sum_{n=1}^{\infty} a_{n}<\infty$, we have

$$
\sum_{n=1}^{\infty}\left(a_{1} a_{2} \cdots a_{n}\right)^{1 / n}<e \sum_{n=1}^{\infty}\left(1-\frac{6}{12 n+11-\frac{5}{12 n}+\frac{17}{90 n^{2}}}\right) a_{n} .
$$

Finally, remark that our new inequalities stated in Theorem 2 can be used to improve the variants of Hardy's inequality established by Zitian and Yibing [9].

\section{REFERENCES}

[1] G. H. Hardy, J. E. Littlewood, and G. Pólya, Inequalities. London: Cambridge Univ. Press, second ed., 1952.

[2] D. S. Mitrinović, Analytic inequalities, ser. Die Grundlehren der mathematischen Wissenschaften. New York-Berlin: Springer-Verlag, 1970.

[3] C. Mortici, "New approximations of the gamma function in terms of the digamma function," Appl. Math. Lett., vol. 23, no. 1, pp. 97-100, 2010.

[4] C. Mortici, "Optimizing the rate of convergence in some new classes of sequences convergent to Euler's constant," Anal. Appl. (Singap.), vol. 8, no. 1, pp. 99-107, 2010.

[5] C. Mortici, "Product approximations via asymptotic integration," Amer. Math. Monthly, vol. 117, no. 5, pp. 434-441, 2010.

[6] D. V. Widder, The Laplace Transform, ser. Princeton Mathematical Series. Princeton, N. J.: Princeton University Press, 1941, vol. 6.

[7] B. Yang, "On Hardy's inequality," J. Math. Anal. Appl., vol. 234, no. 2, pp. 717-722, 1999.

[8] B. Yang and L. Debnath, "Some inequalities involving the constant $e$, and an application to Carleman's inequality," J. Math. Anal. Appl., vol. 223, no. 1, pp. 347-353, 1998.

[9] X. Zitian and Z. Yibing, "A best approximation for constant $e$ and an improvement to Hardy's inequality," J. Math. Anal. Appl., vol. 252, no. 2, pp. 994-998, 2000.

Author's address

Cristinel Mortici

Valahia University of Târgovişte, Department of Mathematics, Bd. Unirii 18, 130056 Târgovişte, Romania

E-mail address: cmortici@valahia.ro 